\title{
Prediction of The Behavior of Bio-Loaded Flexible PVC by Chicken Feathers with Artificial Neural Networks
}

\author{
Abdelghani Lakhdar ${ }^{1}$, Aziz Moumen ${ }^{1}$, Zineb Laabid $^{1}$, and Khalifa Mansouri ${ }^{1}$ \\ ${ }^{1}$ SSDIA Laboratory, Hassan II University of Casablanca, ENSET of Mohammedia, 28830, Morocco
}

\begin{abstract}
The infinite needs of humanity in several fields give birth to several innovations, in materials sciences, these needs are summed up in the creation of new composite materials. Obtaining a composite material from recycled material and a bio-load first of all makes it possible to recover the recycled material and to have new composite material. PVC is one of the most used plastics in recent years, the addition, after recycling, of a bio-load in the form of chicken feathers which are just lost in nature or incinerated, allows the birth of new composite material. In this article, we use neural networks which appear to be among the essential methods to solve and model complex systems, and in particular when it comes to non-linear problems. This method will be used in comparison with the finite element method to find the most adequate method which makes it possible to better model the behaviors of PVC bio-loaded by chicken feathers, and then predict the behaviors with different percentages of the bio-load. We took the results of the experiments carried out, with those of the study of finite elements, and with the results obtained by the neural networks which will be presented in this article, to find the model which makes it possible to better present and predict the behavior of the recycled PVC bio-loaded with an adequate percentage which improves the mechanical characteristics of this type of composite material. The error between the experimental results and those obtained by the neural networks is very small compared to the differences between the two experimental results and those obtained by the finite elements. which proves that the neural network model is an important computer tool for modeling and predicting the results of mechanical experiments
\end{abstract}

\section{Introduction}

The study on composite materials requires before experimental tests, a numerical modeling to study the mechanical, thermal, electrical and other behaviors. Numerical modeling by a computer tool allows flexibility in the change of inputs to visualize the behavior before the performance of the experiment.

In this article, we used two numerical modeling methods, the finite element method already treated [1-4] and we will just take the results of this one, and the neural networks.

Using very powerful tools and algorithms, neural networks are particularly applied to solve problems of [5-9]:

$\checkmark$ Prediction,

$\checkmark$ Classification,

$\checkmark$ Categorization,

$\checkmark$ Optimization,

$\checkmark$ Pattern recognition

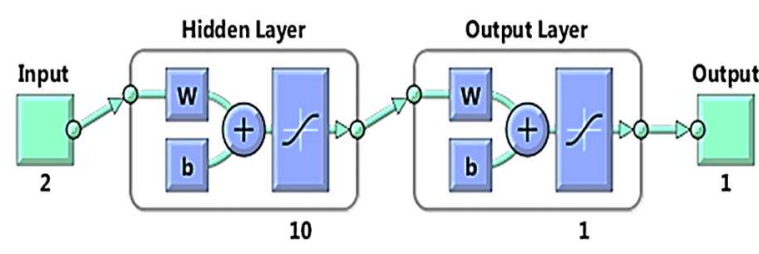

Fig.1. Structure of neural networks by Matlab

In the context of computer science, neural networks constitute a method of approximating complex systems, particularly useful when these systems are difficult to model by classical statistical methods. Neural networks are also applicable in all situations where there is a nonlinear relationship between the variables.

Over the past ten years, the use of neural networks has developed in many disciplines:

$>$ Prediction of the physico-chemical parameters of the media

$>$ aquatic,

$>$ Climate forecast,

$>$ Forecasting time series in finance.

$>$ Improving industrial production,

$>$ Medical diagnosis

$>$ Security,

$>$ Etc.

\footnotetext{
* Corresponding author : lakhdarabdelghani11@gmail.com
} 
Most plastics are recyclable materials, the recycling of these materials allow them to be valued, they are given a new life, the recycling process degrades the properties of the recycled materials. In this study we seek to improve flexible PVC after its degradation by the recycling process.

The flexible PVC recovered after recycling in this study is obtained by adding chicken feathers as bio-load available everywhere [10-13], as shown in figure 2 below.

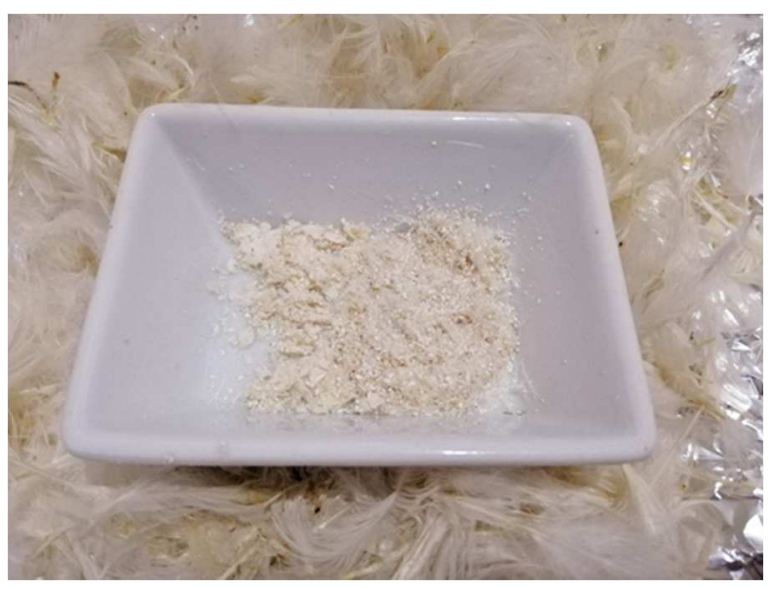

Fig. 2. Chicken feather waste [10]

According to an experimental study and a numerical modeling carried out by finite elements of the addition of three percentages of feathers waste from chickens on flexible PVC, we will attempt a new computer modeling by neural networks, then compare the different studies to find the most suitable model which allows a good prediction of the behavior of flexible bio-loaded PVC.

\section{Experimental Results}

In the experimental study, tensile tests were performed by the EZ200 machine [14] shown in Figure 3 below on samples of flexible PVC bio-loaded with three percentages of chicken feather powder.

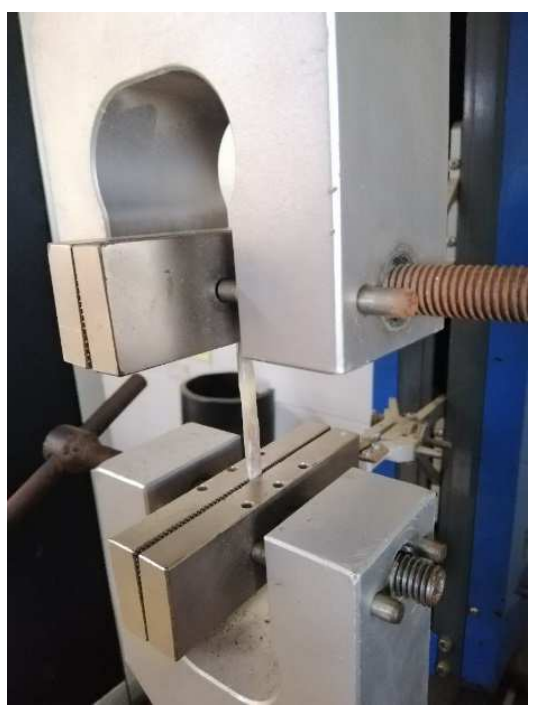

Fig. 3. Tensile test machine
Figure 4 below represents the results of the tests carried out on the three samples bio-loaded successively with 5,10 and $15 \%$ of chicken feathers as well as on a sample of unfilled flexible PVC. The results show different effects of these loads on the mechanical behavior of flexible PVC, in particular in terms of flexibility rigidity. The table 1 summarizes these values concerning the stiffness and elongation at break.

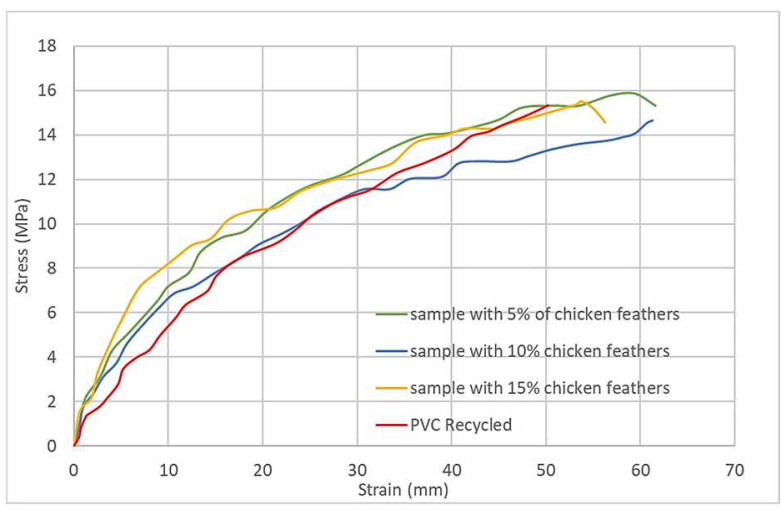

Fig. 4. Flexible PVC with \& without bio-load

Table 1. Stress and strain at break of different samples

\begin{tabular}{|l|c|c|}
\hline & $\begin{array}{c}\text { Stress at } \\
\text { break (MPa) }\end{array}$ & $\begin{array}{c}\text { Strain at } \\
\text { break } \\
(\mathrm{mm})\end{array}$ \\
\hline $\begin{array}{l}\text { Flexible PVC recycled } \\
\text { without bio-loads }\end{array}$ & 15,32 & 50,23 \\
\hline $\begin{array}{l}\text { Flexible PVC bio-loaded } \\
\text { with 5\% of chicken feathers }\end{array}$ & 15,31 & 61,56 \\
\hline $\begin{array}{l}\text { Flexible PVC bio-loaded } \\
\text { with 10\% of chicken } \\
\text { feathers }\end{array}$ & 14,65 & 61,25 \\
\hline $\begin{array}{l}\text { Flexible PVC bio-loaded } \\
\text { with 15\% of chicken } \\
\text { feathers }\end{array}$ & 14,56 & 56,25 \\
\hline
\end{tabular}

\section{Finite Elements Results}

After a random distribution of the bio-load in the flexible PVC matrix, shown in Figure 5, and a triangular mesh as shown in Figure 6, the results of the stress \& strain obtained by the finite element model [15-16] on 4 samples, three are bio-loaded with 5, 10 and 15\% chicken feather waste powder, and one sample without bio-loading, is shown in Figure 7, which shows the different effects of the bio-load on the mechanical behavior of flexible PVC. Table 2 also summarizes the various values obtained by this digital modeling obtained by the finite elements.

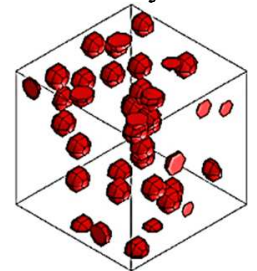

a)

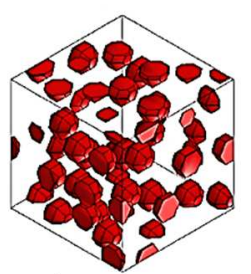

b)

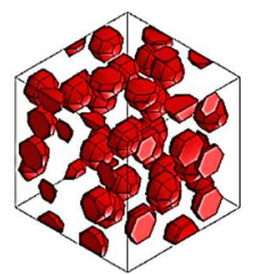

c) 
Fig. 5. Different percentages of chicken feathers distributed in flexible PVC matrix. a) PVC bio-loaded by 5\% chicken feathers; b) PVC bio-loaded by $10 \%$ chicken feathers; c) PVC bio-loaded by $15 \%$ chicken feathers.

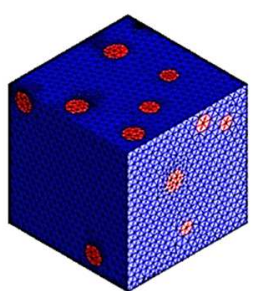

a)

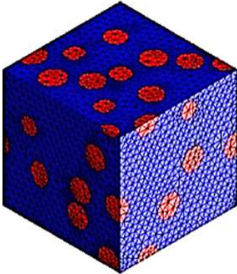

b)

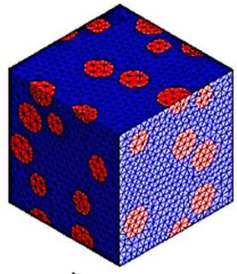

c)
Fig. 6. Mesh of bio-loaded PVC by different percentages. a) PVC bio-loaded by 5\% chicken feathers; b) PVC bio-loaded by $10 \%$ chicken feathers; c) PVC bio-loaded by $15 \%$ chicken feathers.

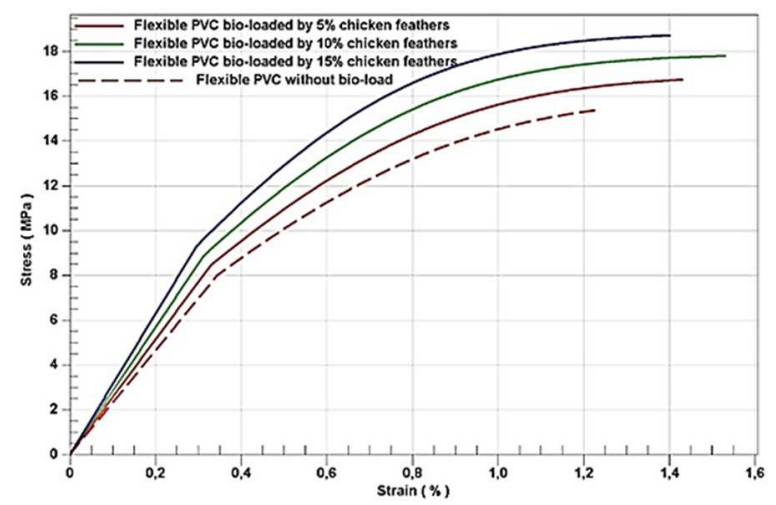

Fig. 7. Tensile test curves of the flexible PVC bio-loaded

\section{Neural Network Study}

\subsection{Presentation of the neural network}

Neural networks, called artificial neural networks are simple imitations of a neuron in the human brain to solve machine learning problems.

The neuron is a unit which is expressed in this study by a hyperbolic Tangent function:

$$
f(x)=\left(2 e^{x} /\left(1+e^{x}\right)\right)-1
$$

In this study, we will use neural networks to predict the behavior of PVC bioloaded by different percentages of chicken feather waste. We will take as input, the values of the stress introduced into the neural network in the form of $21 \times 10,21$ elements for each of 10 test specimen of flexible PVC bioloaded by feathers, and as a target, the experimentally found values of the stress, also in the form of 21 elements corresponds to the mean value.

Figure 8 shows us the global diagram of the shape of the neural network used in this study with the 'TANSIG' as the hidden layer function and the 'PURELIN' function for the output layer

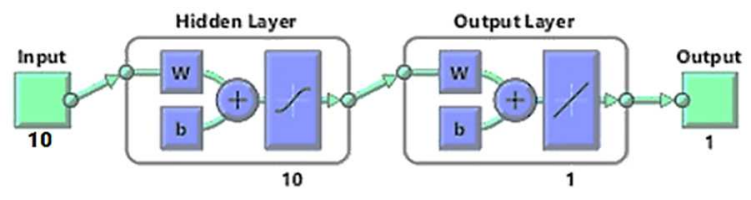

Fig. 8. Neural network diagram

The parameters of this neural network that we have built must be the following:

$>75 \%$ for training per instruction, (mynet.divideparam.trainration $=0.70$; $)$

$>15 \%$ for the test, (mynet.divideparam.testation $=0.15 ;$;

$>15 \%$ for validation, (mynet.divideparam.valration $=0.15 ;$ )

$>1000$ as number of iterations, (mynet.trainparam.epochs $=1000$;)

$>$ The goal must be around $1 \mathrm{e}^{-10}$, (mynet.trainparam.goal = 1e-10;)

\subsection{Neural Network Results}

In this part we will discuss the results of each sample starting with the sample without bio-load of chicken feather waste, Figure 9 shows the performance with the error which is of the order of 0.06256 , as long as this value tends towards 0 we will have a good training and that we will have good results.

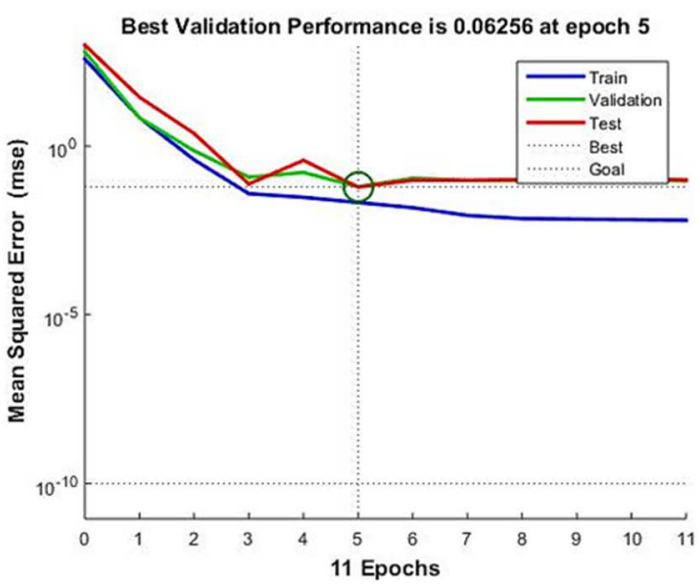

Fig. 9. Validation performance curves of flexible PVC without bio-load

The regression of figure 10 shows the result against the target of the learning, testing, validation curves and all of these last three in the same curve. As long as the value of $\mathrm{R}$ is close to 1 , it means that the results obtained from the neural network with this training correspond to the desired goal, and therefore we have a good training of the network which gives us exactly the desired result. 

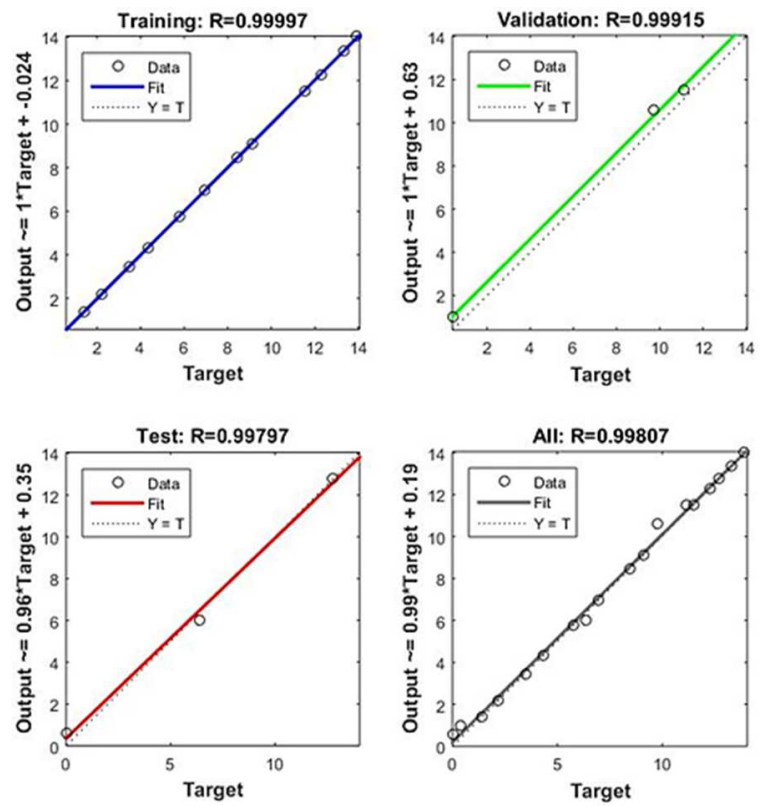

Fig. 10. Regression curves of flexible PVC without bio-load

The two curves represented in figure 11 show the difference between the results obtained by the neural network and that obtained experimentally, in what follows we will take these results again and we will compare them with the results obtained by a study of the finite elements to find the model as close as possible to experiences.

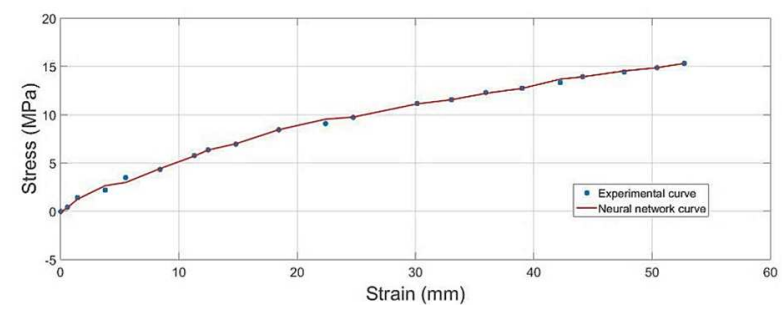

Fig. 11. Experimental and neural network curves of flexible PVC without bio-load

In the figure above, it is clear that the results obtained by the neural networks coincide with the results obtained by the experimental study. We then present the results of the neuron network study on the different samples bio-loaded with 5.10 and $15 \%$ of chicken feather waste.

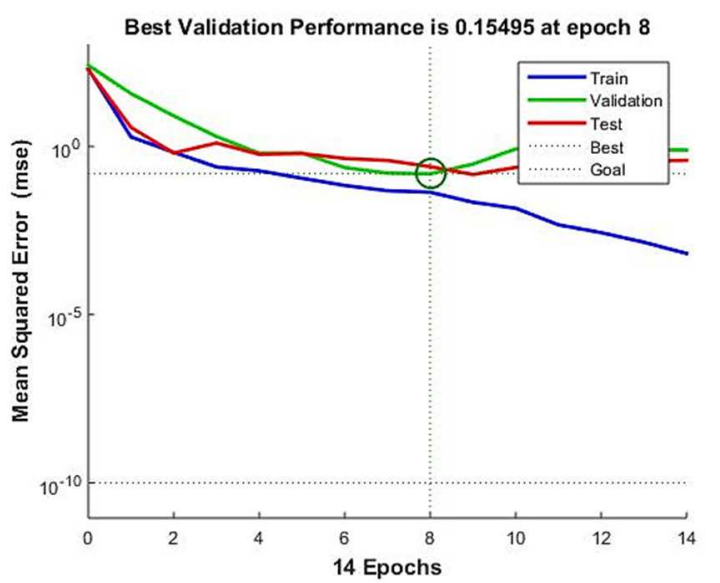

Fig. 12. Validation performance curves of flexible PVC bioloaded by $5 \%$ of chicken feathers

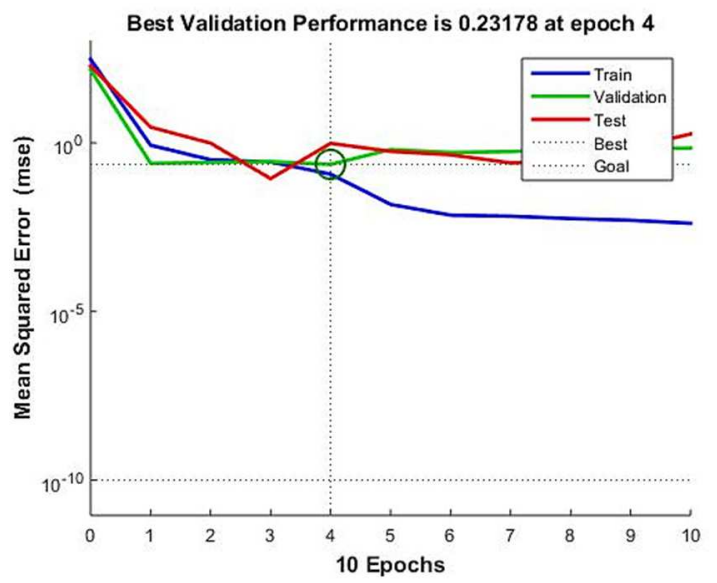

Fig. 13. Validation performance curves of flexible PVC bioloaded by $10 \%$ of chicken feathers

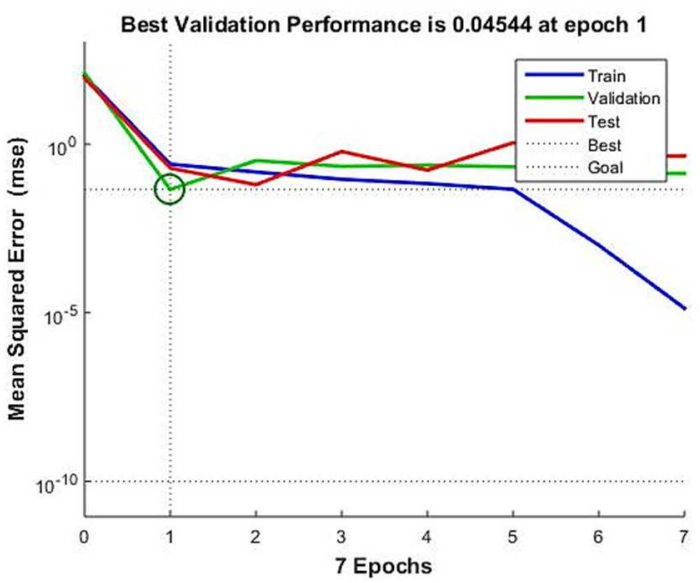

Fig. 14. Validation performance curves of flexible PVC bioloaded by $15 \%$ of chicken feathers

Figures 12,13 and 14 , show the neural network training curves on the $70 \%$ of the inputs with $15 \%$ for the validation and same value for the test, with approximations of the errors for the study on each sample, the errors must be the closest to 0 but, the knowledge of the error alone is not enough to say that we have arrived at a good training, which will lead to good results. We must also study the regression which 
give the error between the output of this study compared to the target described in the following figures
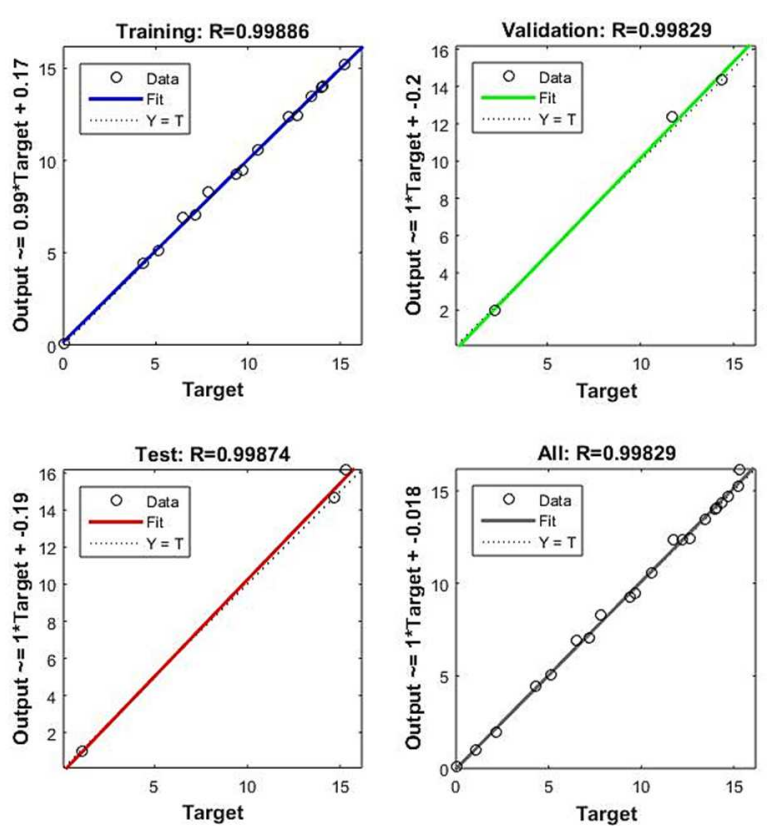

Fig. 15. Regression curves of flexible PVC bio-loaded by $5 \%$ of chicken feathers
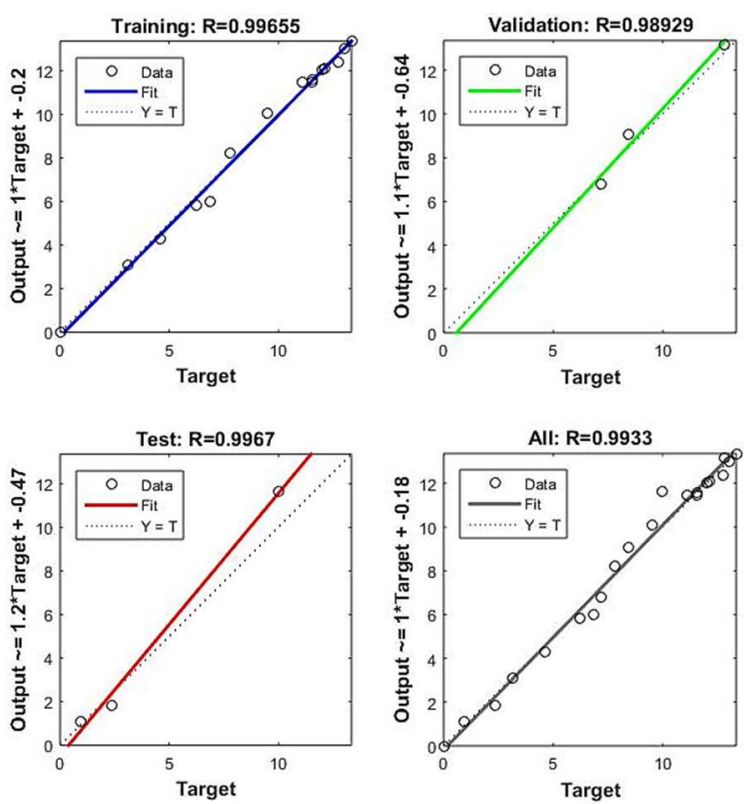

Fig. 16. Regression curves of flexible PVC bio-loaded by $10 \%$ of chicken feathers
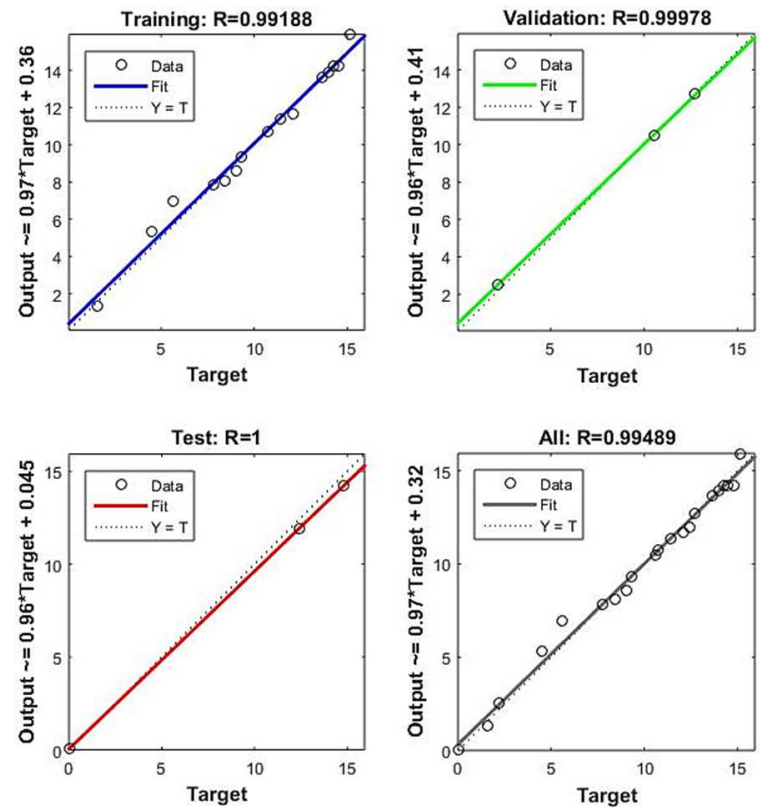

Fig. 17. Regression curves of flexible PVC bio-loaded by $15 \%$ of chicken feathers

Figures 15, 16 and 17 represent the output relative to the target, for the error to be zero, the curve must be a straight line with a slope equal to 1 , and in these three figures we notice that the slopes of the training, testing and validation are all grade 1 , with slight deviations.

\section{Results and Discussion (comparison between finite elements and neural networks)}

The numerical tool makes it possible to model a complex problem to validate and predict results for mainly non-linear problems. in this study we try to predict the behavior of flexible PVC bio-loaded with different percentages by neural networks and to compare these results with the results obtained by the finite elements. The following figures represent the different experimental, finite elements and neural networks results.

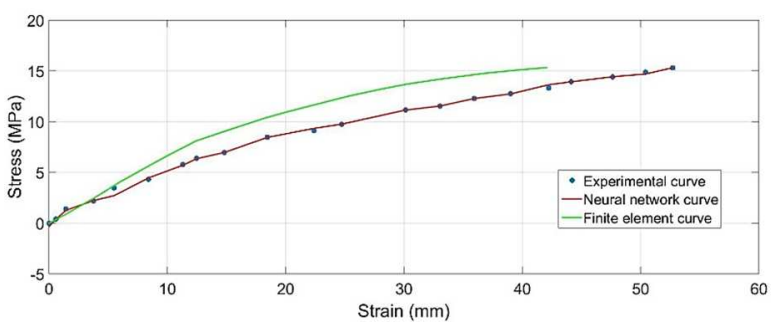

Fig. 18. Experimental, neural network and finite elements curves of flexible PVC without bio-load 


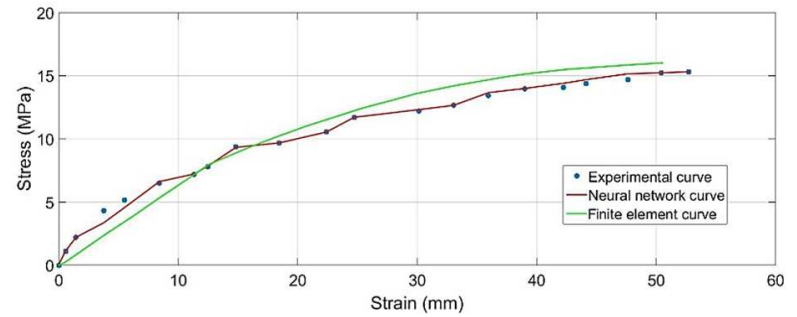

Fig. 19. Experimental, neural network and finite elements curves of flexible PVC bio-loaded by 5\% of chicken feathers

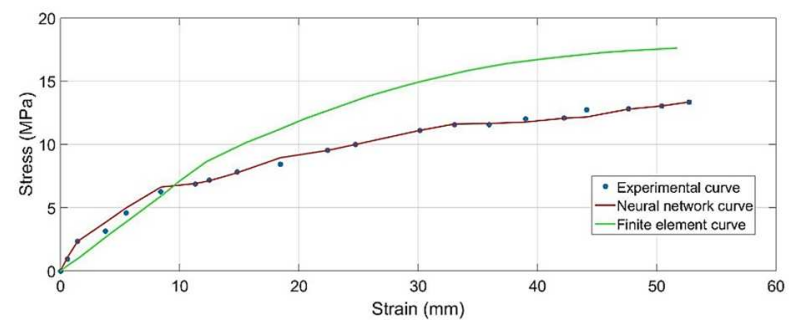

Fig. 20. Experimental, neural network and finite elements curves of flexible PVC bio-loaded by $10 \%$ of chicken feathers

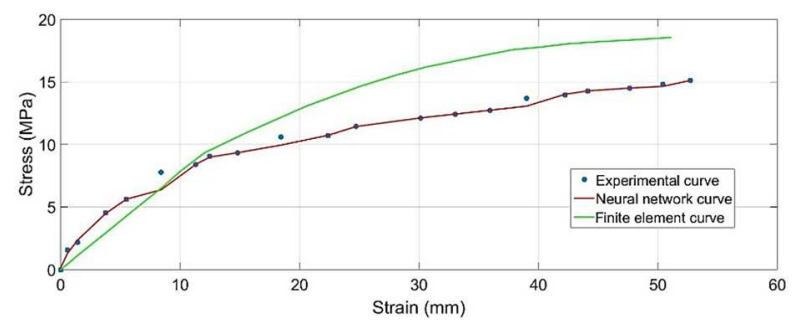

Fig. 21. Experimental, neural network and finite elements curves of flexible PVC bio-loaded by $15 \%$ of chicken feathers

The results represented in figures 18 to 21 , show the difference between finite element modeling and by the study of neural networks.

In the elastic part of the curves the two models give the same results, but in the part elastoplastic the results of the modeling by neural networks coincide with those of the experiments, while the data obtained by the finite elements diverge in this elastoplastic zone.

The study is carried out on four samples, one is not loaded, and three others bio-loaded by 5, 10 and $15 \%$ of chicken feather waste as shown in the figures above, and in each figure, we draw the same remark, that neural networks give good modeling compared to finite elements.

\section{Conclusion}

Numerical modeling and by its computer tool, becomes an essential tool for the modeling and the study of complex problems, and in particular of non-linear problems.

In this study, we modeled the behavior of flexible bio-charged PVC by two models, finite elements and neural networks.

For the finite elements we need the Young's modulus and the Poisson coefficient of the matrix and of the bioload to have the results, whereas these mechanical characteristics of the bio-load used in this study are difficult to find standardized, because they vary from study to study.

For the neural networks we have carried out a training on $70 \%$, tests on $15 \%$ and validations on the last $15 \%$ of the inputs of this network until the good results are found.

The study carried out by neural networks gives a good prediction of the behavior of the new composite material in the two elastic and plastic zones compared to that carried out by the finite elements.

The use of neural networks does not stop here, but it will serve to predict the behavior of flexible PVC bio-loaded by other bio-fillers, as well as to predict the behavior of the flexible PVC matrix bio-loaded by a percentage that we have not experimented with.

\section{References}

1. A. Lakhdar, A. Moumen, L. Zahiri, M. Jammoukh, Kh Mansouri, Experimental and Numerical Study of the Mechanical Behavior of Bio-Loaded PVC Subjected to Aging, Adv. Sci. Technol. Eng. Syst. J, ASTESJ, 5, pp. 607-612, (2020).

2. A. Lakhdar, A. Moumen, Kh. Mansouri, Study of The Mechanical Behavior of Bio Loaded Flexible Pvc by Coconut and Horn Fibers Subjected to Aging, International Journal on Technical and Physical Problems of Engineering, (IJTPE), Iss. 46, 13, No. 1, Mar. (2021)

3. A. Moumen, M. Jammoukh, L. Zahiri, K. Mansouri, Numerical modeling of the thermo mechanical behavior of a polymer reinforced by horn fibers, International Journal of Advanced Trends in Computer Science and Engineering, ASTESJ, 9 , 6541-6548, (2020)

4. P. Marimuthu, C. Kumar, Finite element modelling to predict machining induced residual stresses in the end milling of hard to machine Ti6Al4V alloy, Periodicals of Engineering and Natural Sciences, 7 , 1-11, (2019)

5. Z. Cao, Y. Dan, Z. Xiong, C. Niu, X. Li, S. Qianand, J. Hu, Convolutional Neural Networks for Crystal Material Property Prediction Using Hybrid Orbital-Field Matrix and Magpie Descriptors, Crystals, 9, 191, (2019)

6. D.J. Scott, P.V. Coveney, J.A. Kilner, J.C.H. Rossiny, N.Mc N. Alford, Prediction of the functional properties of ceramic materials from composition using artificial neural networks, Journal of the European Ceramic Society, 27 , 4425-4435, (2007)

7. C. Yang, Y. Kimb, S. Ryu, G. X. Gua, Prediction of composite microstructure stress-strain curves using convolutional neural networks, Materials and Design, 189, (2020)

8. Q. Sun, T. Ertekin, Screening and optimization of polymer flooding projects using artificial-neuralnetwork (ANN) based proxies, J. Pet. Sci. Eng., 185, p. 106617, (2020) 
9. J. S. Chohan, Mechanical Strength Enhancement of $3 D$ Printed Acrylonitrile Butadiene Styrene Polymer Components Using Neural Network Optimization Algorithm, Polymers, BASEL, 12, no. 10 , p. 2250, (2020)

10. A. Lakhdar, A. Moumen, K. Mansouri, Recycled $P V C$ with chicken feathers as bio-load, IOP Conference Series Materials Science and Engineering, India, March (2021)

11. A. Ansarullah, R. Ramli, A. Kusno,B. Baharuddin, N. Jamala, Utilization of waste of chicken feathers and waste of cardboard as the material of acoustic panel maker, Earth and Environmental Science, 1$8,(2018)$

12. I. Aranberri, S. Montes, I. Azcune, A. Rekondo, H-J. Grande, Fully Biodegradable Biocomposites with High Chicken Feather Content, Polymers , 9, 593, (2017)

13. W.F. Schmidt, Innovative Feather Utilization Strategies, in Poultry Waste Management Conference, Springdale, Arkansas (1998)

14. A. Lakhdar, M. Jammoukh, L. Zahiri, K. Mansouri, A. Moumen, B. Salhi, Numerical and Experimental Study of the Behavior of PVC Material Subjected to Aging, 1st International Conference on Innovative Research in Applied Science, Engineering and Technology (IRASET), IEEE: 1-6, (2020)

15. A., Moumen, A., Lakhdar, A., Mansouri, K. Numerical study of the mechanical behavior of polyamide 66 reinforced by argan nut shell particles with the finite element method and the mori-tanaka model, Int. J. Adv. Trends Comput. Sci. Eng., 9, no. 5, pp. 7723-7730, (2020)

16. J. Naveen, M. Jawaid, A. Vasanthanathan, M. Chandrasekar, Finite element analysis of natural fiber-reinforced polymer composites. in Modelling of Damage Processes in Biocomposites, FibreReinforced Composites and Hybrid Composites, Elsevier, pp. 153-170, (2019) 